Med Klin Intensivmed Notfmed 2020 115:390-392 https://doi.org/10.1007/s00063-020-00677-0 Online publiziert: 26. März 2020

(c) Springer Medizin Verlag GmbH, ein Teil von Springer Nature 2020

\section{Guido Michels ${ }^{1} \cdot$ Uta Ochmann ${ }^{2} \cdot$ Rita Cranen ${ }^{1}$}

${ }^{1}$ Klinik für Akut- und Notfallmedizin, St.-Antonius-Hospital gGmbH, Akademisches Lehrkrankenhaus der RWTH Aachen, Eschweiler, Deutschland

${ }^{2}$ Institut und Poliklinik für Arbeits-, Sozial- und Umweltmedizin, Stabsstelle Betriebsärztlicher Dienst, LMU Klinikum, München, Deutschland

\title{
Mutterschutz - auch im Zeitalter der Coronakrise
}

Die Infektion mit dem Coronavirus SARS-CoV-2 breitet sich nicht nur national sondern auch weltweit zunehmend aus, sodass die SARS-CoV-2-Infektion am 11.03.2020 von der Weltgesundheitsorganisation (WHO) zur Pandemie erklärt wurde. Gerade in endemischen Krisengebieten nehmen zum einen die Zahl der SARS-CoV-2-Fälle tagtäglich zu und zum anderen die Zahl der SARSCoV2-bestätigten Mitarbeiter und damit auch die Kontaktpersonen der Kategorie I mit engem Kontakt stetig zu. Damit nicht genug, denn auch die Anzahl schwerer Krankheitsverläufe wird zunehmend steigen, sodass mit einem steigenden Bedarf an Intensiv- und Beatmungskapazitäten zur Behandlung von an Coronavirus erkrankten (COVID-) Patienten zu rechnen ist. Die Betreuung dieser Patienten setzt jedoch eine stabile personelle Besetzung durch pflegerische und ärztliche Mitarbeiter voraus. Zur Schaffung einer stabilen personellen Versorgungsstruktur wurde für Pflegekräfte auf Intensivstationen vor Monaten die Pflegepersonaluntergrenzenverordnung (PpUGV) geschaffen, die jedoch unter den Aspekten des Krisenmanagements außer Kraft gesetzt wurde. Gerade Kontaktpersonen der Kategorie I sind durch ein hohes Infektionsrisiko gekennzeichnet und sollten eine häusliche Absonderung einhalten. Besonders Kliniken in betroffenen Gebieten in Deutschland (z.B. Landkreis Heinsberg/NordrheinWestfalen) weichen - um die Versorgung der Bevölkerung sicherzustellen schon seit Wochen von der empfohlenen Vorgehensweise des Robert KochInstituts (RKI) ab. Asymptomatische
Mitarbeitende in Krankenhäusern, die Kontaktpersonen der Kategorie I sind, sollen zur Patientenversorgung weiter zur Verfügung stehen. Lediglich das Tragen eines Mund-Nasen-Schutzes bei Patientenkontakt, das Führen eines Gesundheitstagebuchs, regelmäßige vorsorgliche Abstrichuntersuchungen und das Einhalten einer Hausquarantäne im Privatleben werden gefordert. Bedingt durch diese multifaktorielle Entwicklung resultiert ein zunehmender Personalengpass auf sämtlichen medizinischen Versorgungsebenen, sodass „jede Mitarbeiterin“ bzw. ,jeder Mitarbeiter" kritischer Infrastrukturen des Gesundheitssystems notwendig ist. Sowohl Mitarbeiter/-innen im pflegerischen als auch im ärztlichen Bereich leisten zurzeit unglaubliches, und arbeiten zum Teil gerade in der Akutmedizin - über ihre Grenzen hinaus. Dabei setzen sie sich, um die medizinische Versorgung weiterhin zu gewährleisten, oftmals großen Gefahren aus, ohne an sich selber zu denken.

Gerade schwangere Mitarbeiterinnen, die neben chronisch lungenkranken, immunsupprimierten und älteren Menschen zur Risikogruppe gehören, sollten in dieser Krisensituation geschützt werden. Basierend auf der Tatsache, dass sich das Immunsystem während der Schwangerschaft unter anderem durch die Exposition gegenüber fetalen Antigenen verändert, sind Frauen einem höheren Infektionsrisiko ausgesetzt. Die meisten Virusinfektionen, z. B. die Influenza-A-Virus-Infektion, sind daher während der Schwangerschaft mit einem schweren Krankheitsverlauf assoziiert
[1]. Daher können neben den InfluenzaA-Viren auch Coronaviren, die für das schwere akute respiratorische Syndrom („severe acute respiratory syndrome“, SARS) und das respiratorische Syndrom des Nahen Ostens (Middle East Respiratory Syndrome, MERS) verantwortlich sind, schwere negative Auswirkungen auf die Schwangerschaft zur Folge haben, wie z.B. Fehlgeburten, Frühgeburten, intrauterine Wachstumseinschränkungen bis hin zum Tod der Mutter [2, 3]. Zum Themenkomplex „CoronavirusSARS-CoV-2-Infektion und Schwangerschaft" liegen aktuell nur wenige Daten vor. In einer kürzlich publizierten retrospektiven Beobachtungsstudie mit 9 schwangeren Patientinnen konnte gezeigt werden, dass es derzeit keine Hinweise auf eine intrauterine Infektion durch vertikale Übertragung bei Frauen gibt, die in der späten Schwangerschaft eine COVID-19-Pneumonie entwickeln [4]. Gegensätzlich von dieser kleinen Studie existiert ein Fallbericht, der ähnlich den Beobachtungen mit SARS - eine vertikale Übertragung von der Mutter auf das Kind vermutet [5].

Da SARS-CoV-2-infizierte Schwangere ein vulnerables Kollektiv darstellen, wurde von den Kollegen Favre et al. eine internationale „Leitlinie für schwangere Frauen mit Verdacht auf SARS-CoV2-Infektion“ vorgeschlagen [6]. Die Autoren empfehlen, dass jede schwangere Frau, die innerhalb der letzten 14 Tage in ein von SARS-CoV-2 betroffenes Risikogebiet gereist ist oder die engen Kontakt zu einem bestätigtem SARS-CoV-2Fall hatte, getestet werden sollte, auch wenn der Verlauf asymptomatisch ist. 
Schwangere Frauen mit einer bestätigten SARS-CoV-2-Infektion, die asymptomatisch sind, sollten für mindestens 14 Tage Hausquarantäne einhalten und ein Gesundheitstagebuch führen. Asymptomatische Schwangere, die Kontaktpersonen der Kategorie I sind, sollten wegen des potenziellen Risikos einer intrauterinen Wachstumsretardierung alle 2 Monate mittels Ultraschalluntersuchung überwacht werden [6].

Das Mutterschutzgesetz bezieht sich auf im Vergleich zum allgemeinen Lebensrisiko erhöhte berufliche Gefährdungen. Im Mutterschutzgesetz wird definitiv ausgeführt, dass Mitarbeiter keinen Kontakt zu Infektiösen haben dürfen, darunter fallen auch Menschen mit SARS-CoV-2-Infektionen, hier bedarf es keiner neuen Regelung. Vor dem aktuellen Hintergrund möchten wir noch einmal auf die wichtigsten Eckdaten des Mutterschutzes am Arbeitsplatz hinweisen:

- Die konsequente Einhaltung der persönlichen Hygienemaßnahmen ist der beste Schutz und wird bei allen Schwangeren empfohlen.

- Der Arbeitgeber soll eine Gefährdungsbeurteilung des Arbeitsplatzes vornehmen (Verpflichtung nach $\$ 5$ Arbeitsschutzgesetz) und hierbei auch die schwangerschaftsbedingten Risiken beurteilen ( $\$ 10$ Mutterschutzgesetz). Darüber hinaus soll der Arbeitgeber der schwangeren Frau ein Gespräch über mögliche weitere Anpassungen der Arbeitsbedingungen anbieten $(\$ 10$ Mutterschutzgesetz). Hierbei ist es empfehlenswert, die Betriebsärztin bzw. den Betriebsarzt einzubinden.

- Grundsätzlich darf der Arbeitgeber eine Schwangere keine Tätigkeiten ausüben lassen, bei der sie mit Biostoffen der Risikogruppe 2, 3 oder 4 gemäß $\$ 3$ Absatz 1 Biostoffverordnung in Kontakt kommt oder kommen kann (SARS-CoV-2 zählt zur Risikogruppe 3 nach Biostoffverordnung) und dies für die Frau oder ihr Kind eine unverantwortbare
Gefährdung darstellt ( $\$ 11$ Abs. 2 Mutterschutzgesetz). Erst wenn geklärt ist, dass eine unverantwortbare Gefährdung ausgeschlossen ist, z. B. durch einen bestehenden Immunbzw. Impfschutz, kann die Schwangere die Tätigkeit fortsetzen.

- Bei Tätigkeiten im Krankenhaus kann es in Abhängigkeit vom Arbeitsplatz und den konkreten Arbeitsbedingungen zu einer erhöhten Infektionsgefährdung kommen, hier sind insbesondere die Intensivstation und die Notaufnahme zu erwähnen. Schwangere dürfen nicht im Erstkontakt zu Patienten eingesetzt werden. Hilfreich kann eine Anpassung der Arbeitsorganisation sein, indem z. B. Patienten, von denen ein Infektionsrisiko ausgehen kann, räumlich getrennt behandelt werden, damit die Schwangere nicht mit ihnen in Kontakt kommen kann. Ist keine Umgestaltung der Arbeitsbedingungen möglich und auch kein Einsatz an einem anderen Arbeitsplatz, müssen

Hier steht eine Anzeige. 
(befristete) betriebliche Beschäftigungsverbote in Betracht gezogen werden.

- Kann eine unverantwortbare Gefährdung am Arbeitsplatz nicht ausgeschlossen werden, sollen die Arbeitsbedingungen entsprechend umgestaltet werden. Ist auch dies nicht möglich, kann die Schwangere ihre Tätigkeit an diesem Arbeitsplatz nicht mehr fortführen.

- Ist keine Umgestaltung der Arbeitsbedingungen möglich und auch kein Einsatz an einem anderen Arbeitsplatz, sollen (befristete) betriebliche Beschäftigungsverbote in Betracht gezogen werden.

- Bei nachgewiesenem Erkrankungsfall durch das SARS-CoV-2-Virus im Arbeitsbereich der Schwangeren sollten Schwangere für 14 Tage freigestellt werden, um sicherzustellen, dass keine weiteren Personen infiziert wurden.

Neben diesen Empfehlungen verweisen wir auf die tagesaktuellen Informationen auf den Seiten des RKI [7].

\section{Korrespondenzadresse}

\section{Prof. Dr. med. Guido Michels}

Klinik für Akut- und Notfallmedizin, St.Antonius-Hospital gGmbH, Akademisches Lehrkrankenhaus der RWTH Aachen Dechant-Deckers-Str. 8, 52249 Eschweiler, Deutschland guido.michels@sah-eschweiler.de
2. Alfaraj SH, Al-Tawfiq JA, Memish ZA (2019) Middle East respiratory syndrome coronavirus (MERS(oV) infection during pregnancy: report of two cases \& review of the literature. J Microbiol Immunol Infect 52:501-503

3. Wong SF, Chow KM, Leung TN et al (2004) Pregnancy and perinatal outcomes of women with severe acute respiratory syndrome. Am J Obstet Gynecol 191:292-297

4. Chen $\mathrm{H}$, Guo J, Wang $\mathrm{C}$, Luo $\mathrm{F}$, Yu X, Zhang W, Li J, Zhao D, Xu D, Gong Q, Liao J, Yang H, Hou W, Zhang Y (2020) Clinical characteristics and intrauterine vertical transmission potential of COVID-19 infection in nine pregnant women: a retrospective review of medical records. Lancet 7;395(10226):809-815 (Mar)

5. Wang S, Guo L, Chen L, Liu W, Cao Y, Zhang J, Feng L. A case report of neonatal COVID-19 infection in China.Clin Infect Dis. 2020 Mar 12. pii: ciaa225.

6. Favre G, Pomar L, Qi X, Nielsen-Saines K, Musso $D$, Baud D. Guidelines for pregnant women with suspected SARS-CoV-2 infection. Lancet Infect Dis. 2020 Mar 3. pii:S1473-3099(20)30157-2.

7. https://www.rki.de/DE/Content/InfAZ/N/ Neuartiges_Coronavirus/nCoV.html
Med Klin Intensivmed Notfmed 2020 - 115: 390-392

https://doi.org/10.1007/s00063-020-00677-0

(c) Springer Medizin Verlag $\mathrm{GmbH}$, ein Teil von Springer Nature 2020

\section{G. Michels · U. Ochmann · R. Cranen \\ Mutterschutz - auch im Zeitalter der Coronakrise}

\section{Zusammenfassung}

Schwangere Mitarbeiterinnen sollten insbesondere in Krisensituationen geschützt werden. Im Mutterschutzgesetz wird ausgeführt, dass Mitarbeiter keinen Kontakt zu Infektiösen haben dürfen, darunter fallen auch Menschen mit SARS-CoV-2 Infektionen; hier bedarf es keiner neuen Regelung.

Schlüsselwörter

Schwangerschaft · Mitarbeiter · Prävention . Coronavirus · Akutmedizin

\section{Maternity protection-also during the corona crisis}

\section{Abstract}

Pregnant employees should be protected, particularly in crisis situations. The Maternity Protection Act states that employees are not allowed to have contact with infectious people, including people with SARS-CoV2 infections; no new regulation is required here.

\section{Keywords}

Pregnancy - Employees · Prevention .

Coronavirus $\cdot$ Acute care medicine

\section{Einhaltung ethischer Richtlinien}

Interessenkonflikt. G. Michels, U. Ochmann und R. Cranen geben an, dass kein Interessenkonflikt besteht.

Für diesen Beitrag wurden von den Autoren keine Studien an Menschen oder Tieren durchgeführt. Für die aufgeführten Studien gelten die jeweils dort angegebenen ethischen Richtlinien.

\section{Literatur}

1. Le Gars M, Seiler C, Kay AW, Bayless NL, Starosvetsky $E$, Moore $L$, Shen-Orr SS, Aziz N, Khatri $P_{\text {, }}$ Dekker CL, Swan GE, Davis MM, Holmes S, Blish CA (2019) Pregnancy-Induced Alterations in NK Cell Phenotype and Function. Front Immunol 23;10:2469 (Oct) 